\title{
Venetoclax and Daratumumab combination treatment demonstrates pre-clinical efficacy in mouse models of Acute Myeloid Leukemia
}

\author{
Jayna J Mistry ${ }^{1,2}$, Charlotte Hellmich ${ }^{1,3}$, Amelia Lambert ${ }^{1}$, Jamie A Moore ${ }^{1}$, Aisha Jibril ${ }^{1}$, Angela Collins ${ }^{3}$, \\ Kristian M Bowles ${ }^{1,3,4^{*}}$ and Stuart A Rushworth ${ }^{1,4^{*}}$
}

\begin{abstract}
Acute myeloid leukemia (AML) remains an incurable malignancy despite recent advances in treatment. Recently a number of new therapies have emerged for the treatment of $A M L$ which target $B C L-2$ or the membrane receptor CD38. Here, we show that treatment with Venetoclax and Daratumumab combination resulted in a slower tumor progression and a reduced leukemia growth both in vitro and in vivo. These data provide evidence for clinical evaluation of Venetoclax and Daratumumab combination in the treatment of AML.
\end{abstract}

To the editor,

Acute Myeloid Leukemia (AML) is associated with poor prognosis and high mortality causing around 85,000 global deaths per year [1]. Current standard treatment for AML is poorly tolerated and most therapy is not curative for patients. The intensive nature of proficient therapeutics for AML are more suited towards younger patients, which is not the typical age-group for this disease. This results in limited effective treatment options, highlighting the need for more targeted, well-tolerated treatments.

Recently numerous novel therapies have emerged for treatment of AML. The BCL-2 family proteins are important in regulating intrinsic apoptotic processes [2]. Suppression of mitochondrial-mediated apoptosis by BCL-2 overexpression is a hallmark of AML progression, often correlated with a poor response to cytotoxic treatment [3, 4]. Recently, clinical trials using BCL-2 inhibition by the $\mathrm{BH} 3$ mimetic Venetoclax has been shown to be effective in

\footnotetext{
* Correspondence: k.bowles@uea.ac.uk; s.rushworth@uea.ac.uk

${ }^{1}$ Norwich Medical School, University of East Anglia, Norwich Research Park, NR4 7UQ Norwich, UK

Full list of author information is available at the end of the article
}

promoting caspase-dependent AML cell death [5], leading to Venetoclax receiving FDA approval for treatment of AML in combination with low dose cytarabine, decitabine or the hypomethylating azacytidine [6]. This is an important milestone for AML treatment, as no curative therapies have shown to be clinically effective at treating this disease.

Unfortunately, AML resistance to Venetoclax is common, therefore, numerous studies combining Venetoclax with other therapies are being trialed [6, 7]. Our group and others have reported CD38 is overexpressed in AML and CD38 inhibition using Daratumumab is effective in preclinical AML models $[8,9]$. CD38 is a transmembrane glycoprotein expressed on many hematopoietic cells [10]. In our recent study we demonstrated that Daratumumab works by inhibiting mitochondrial transfer from mesenchymal stromal cell (MSC) to AML blasts in the bone marrow (BM). Here we hypothesized that inhibiting the CD38 using daratumumab, in combination with BCL-2 inhibition using Venetoclax, could be a strategy to improve overall survival of AML patients.

To investigate BCL-2 inhibition in combination with CD38 inhibition in AML we used primary AML blasts 
isolated from patients BM (Table 1). CD38 and BCL-2 expression on isolated AML and non-malignant control CD34 cells were measured by flow cytometry (Fig. 1 a and supplementary Figure 1). Cell viability was assessed using various doses of Venetoclax or Daratumumab and showed that $100 \mathrm{nM}$ Venetoclax alone inhibited AML cell viability (Fig. 1b). Daratumumab alone had no effect on cell viability at any concentration used (Fig. 1b). Furthermore, in combination with Venetoclax, Daratumamab had no additive effect on AML survival (Fig. $1 \mathrm{c}$ ). Since AML is highly reliant on the BM microenvironment, we used an in vitro coculture system, AML was cultured with MSC with either Venetoclax alone, Daratumumab alone, or Venetoclax and Daratumumab combination for $24 \mathrm{~h}$. AML cells were then stained with AnnexinV-FITC/PI and analyzed using flow cytometry. Cells treated with Venetoclax and
Daratumumab combination had significantly higher apoptosis compared to untreated AML cells (Fig. 1d and e). Treatment with Venetoclax or Daratumumab alone had no significant effect on AML apoptosis when cultured under these conditions, suggesting that these targeted therapies are working through different pathways. Together, these results highlight the need to study the effectiveness of Venetoclax and Daratumumab combination in in vivo models of AML.

To do this we used a NOD.Cg-Prkdcscid IL2rgtm1Wjl/SzJ (NSG) xenograft mouse model of AML whereby on day 1 we transplanted $0.5 \times 10^{6} \mathrm{MV} 411$ cells or patient derived AML tagged with a luciferase construct into the tail vein of NSG mice. On day 7 we analyzed NSG mice for AML engraftment. Mice were split into treatment groups including vehicle control (PBS),

Table 1 AML diagnostic information

\begin{tabular}{|c|c|c|c|c|c|c|c|c|}
\hline $\begin{array}{c}\text { Numbe } \\
r\end{array}$ & Age & Sex & Diagnosis & $\begin{array}{l}\text { Molecular } \\
\text { Karyotype }\end{array}$ & $\begin{array}{l}\text { Molecular } \\
\text { genetics }\end{array}$ & $\begin{array}{c}\text { CD38 } \\
\mathrm{MFI}\end{array}$ & $\begin{array}{c}\mathrm{BCL}-2 \\
\mathrm{MFI}\end{array}$ & symbol \\
\hline 1 & 61 & $M$ & $\begin{array}{c}\text { Acute myeloid } \\
\text { leukaemia with a } \\
\text { monocytic component }\end{array}$ & $46 \mathrm{XY}$ & \begin{tabular}{|c|} 
FLT3 internal tandem \\
duplication; NPM1 \\
exon 12 mutation : \\
CEBPA mutation : \\
Pathogenic variants \\
in DNMT3A and \\
NPM1
\end{tabular} & & & \\
\hline 2 & 74 & $M$ & \begin{tabular}{|c|} 
Acute myeloid \\
leukaemia with \\
myelodysplasia related \\
changes
\end{tabular} & Complex & $\begin{array}{c}\text { No mutations in Flt3, } \\
\text { NPM1 exon } 12 \text { or } \\
\text { CEBPA }\end{array}$ & 42 & 43 & \\
\hline 3 & 68 & $M$ & $\begin{array}{c}\text { Acute myeloid } \\
\text { leukaemia with two FLT- } \\
3 \text { ITDs detected with a } \\
\text { high allelic ratio (FLT3- } \\
\text { ITDhigh). }\end{array}$ & $46 X Y$ & $\begin{array}{l}\text { FLT3-ITDhigh; no } \\
\text { mutations in NPM1 } \\
\text { exon } 12 \text { or CEBPA }\end{array}$ & 55 & 73 & \\
\hline 4 & 59 & $\mathrm{M}$ & \begin{tabular}{|c|} 
Acute myeloid \\
leukaemia with \\
myelodysplasia related \\
changes
\end{tabular} & Complex & \begin{tabular}{|c|} 
No mutations in Flt3, \\
NPM1 exon 12 or \\
CEBPA
\end{tabular} & 58 & 102 & \\
\hline 5 & 54 & $\bar{F}$ & $\begin{array}{c}\text { Acute myeloid } \\
\text { leukaemia with MLL } \\
\text { (11q23) rearrangement }\end{array}$ & \begin{tabular}{|c|} 
Tetrasomy 8 and \\
$\mathrm{t}(9 ; 11)(\mathrm{p} 21.3 ; \mathrm{q} 23$ \\
$.3) ; \mathrm{KMT} 2 \mathrm{~A}-$ \\
MLLT3
\end{tabular} & No mutations in Flt3 & & & \\
\hline 6 & 59 & $M$ & \begin{tabular}{|c|} 
Relapsed Relapse of \\
Acute myeloid \\
leukaemia, with bi- \\
allelic CEBPA mutations
\end{tabular} & Complex & \begin{tabular}{|c|} 
Pathogenic variants \\
detected in DNMT3A, \\
GATA2 and \\
CEBPA(two variants)
\end{tabular} & 92 & 79 & \\
\hline 7 & 55 & $\mathrm{~F}$ & $\begin{array}{c}\text { Acute myeloid } \\
\text { leukaemia with } \\
\text { myelodysplasia related } \\
\text { changes }\end{array}$ & $\begin{array}{l}\text { Monosomy 7; } \\
\text { Trisomy } 8\end{array}$ & \begin{tabular}{|c|} 
Pathogenic variants \\
in RUNX1, SRSF2, \\
ASXL1 and TET2; No \\
mutations in Flt3, \\
NPM1 exon 12 or \\
CEBPA
\end{tabular} & 98 & 54 & \\
\hline 8 & 75 & $\mathrm{~F}$ & \begin{tabular}{|c|} 
Acute myeloid \\
leukaemia with \\
myelodysplasia related \\
changes
\end{tabular} & Trisomy 8 & $\begin{array}{c}\text { No mutations in Flt3, } \\
\text { NPM1 exon } 12 \text { or } \\
\text { CEBPA }\end{array}$ & 43 & 48 & \\
\hline 9 & 37 & $M$ & $\begin{array}{c}\text { Acute myeloid } \\
\text { leukaemia without } \\
\text { maturation }\end{array}$ & $46 X Y$ & $\begin{array}{c}\text { No mutations in Flt } 3, \\
\text { NPM1 exon } 12 \text { or } \\
\text { CEBPA }\end{array}$ & & & \\
\hline
\end{tabular}




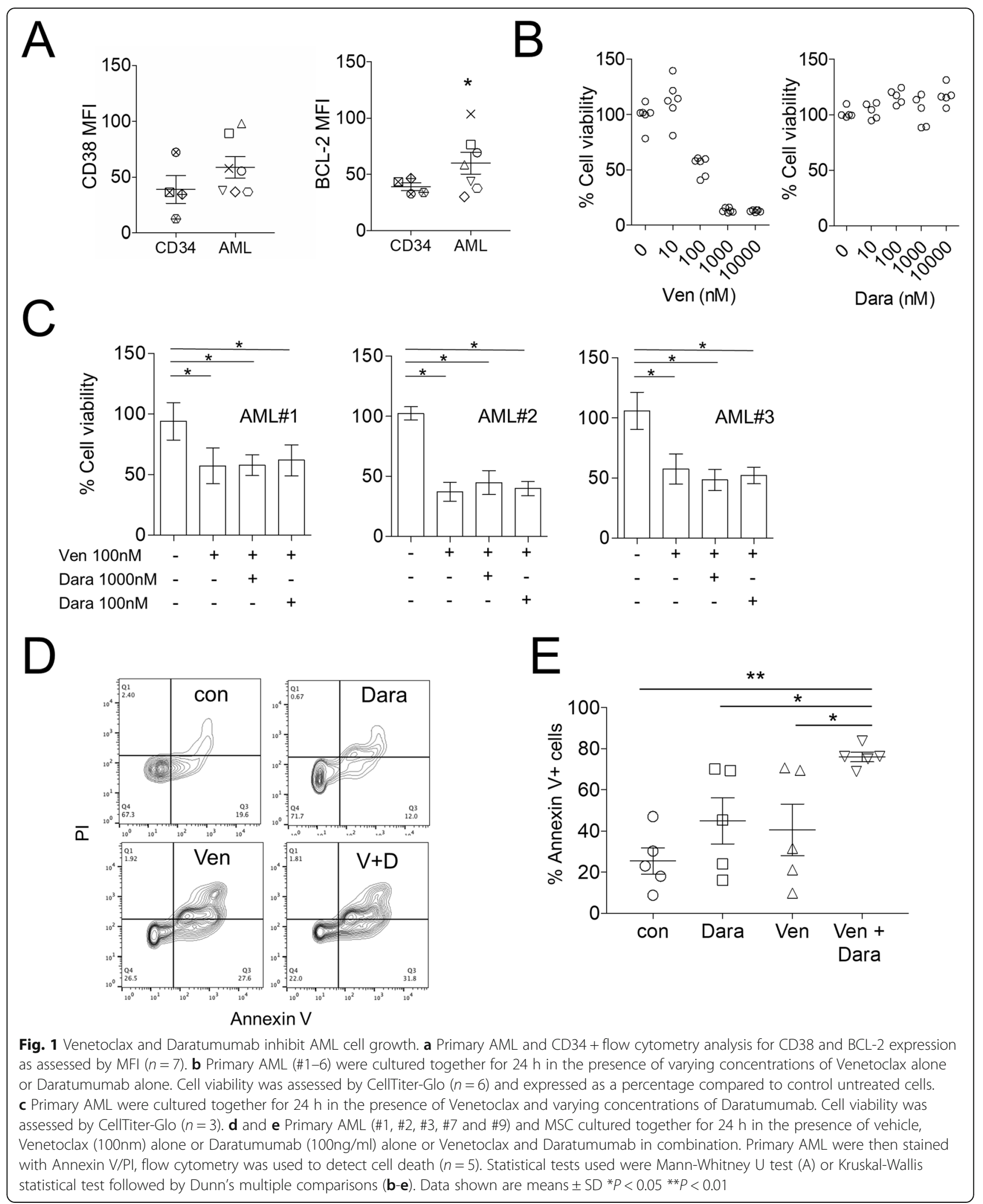

Daratumumab alone on day 7 and 14 by intraperitoneal injection $(5 \mathrm{mg} / \mathrm{kg})$, Venetoclax alone by oral gavage (100 $\mathrm{mg} / \mathrm{kg} /$ day) for 10 days or combination
Daratumumab $(5 \mathrm{mg} / \mathrm{kg})$ and Venetoclax $(100 \mathrm{mg} / \mathrm{kg} /$ day) (Fig. 2 a). The mice were then imaged using live in vivo bioluminescence imaging on day 17 to determine 


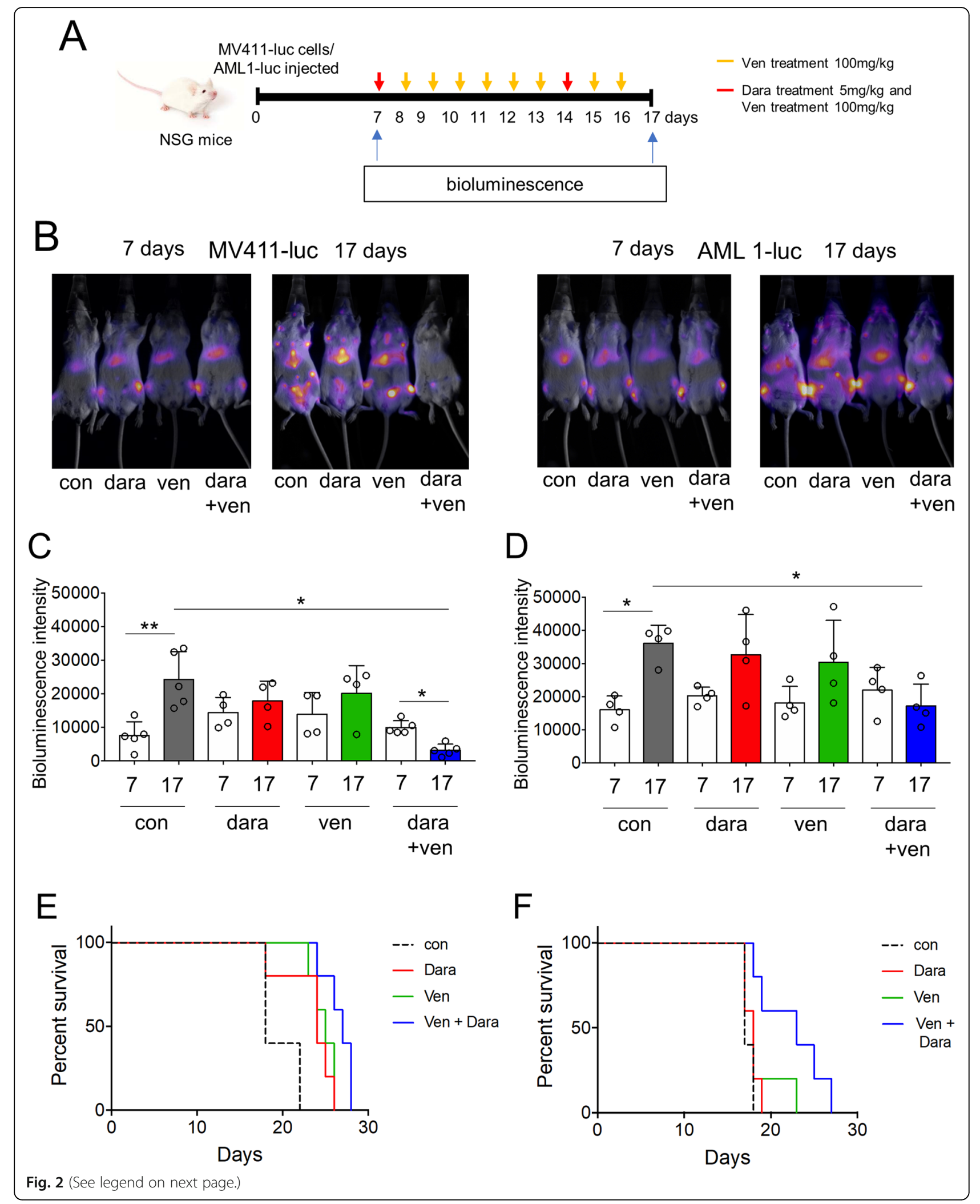


(See figure on previous page.)

Fig. 2 Venetoclax and Daratumumab combination inhibits AML disease progression in vivo. a Schematic of the in vivo model used for these experiments. $\mathbf{b} 0.5 \times 10^{6} \mathrm{MV} 411$ cells or primary AML (\#3) were injected into the tail vein of NSG mice. Mice were imaged using bioluminescence at day 7 following injection to confirm tumor engraftment, and then split into four groups. Group 1 received vehicle (PBS), group 2 received Daratumumab (Dara; $5 \mathrm{mg} / \mathrm{kg}$ ) on day 7 and day 14 by intra-peritoneal (IP) injection, group 3 received Venetoclax (Ven; $100 \mathrm{mg} / \mathrm{kg}$ ) daily by oral gavage (OG) and group 4 received Venetoclax (Ven; $100 \mathrm{mg} / \mathrm{kg}$ ) daily by OG and Daratumumab (Dara; $5 \mathrm{mg} / \mathrm{kg}$ ) on day 7 and day 14 by IP. Mice were then imaged using bioluminescence at day 17. $\mathbf{c}$ and $\mathbf{d}$ Densitometry of the bioluminescent images shown in (B) was performed to determine differences between vehicle, Daratumumab alone, Venetoclax alone or Daratumumab and Venetoclax treated animals. $(n>4)$. Statistical tests used was Kruskal-Wallis statistical test followed by Dunn's multiple comparisons. Data shown are means \pm SD $* P<0.05 * * P<0.01$. ( $\mathrm{E}$ and F) Kaplan-Meier survival curves of treatment groups

tumor burden (Fig. 2b). The densitometry measurement of treated animals and survival graphs confirmed combination Daratumumab and Venetoclax was the most effective at reducing tumor burden (Fig. 2 c-f). Given the heterogeneity of AML, one limitation of the study is that only one AML patient sample and one cell line was used in the in vivo experiments. Finally, in phase $1 / 2$ clinical trials in relapse/refractory myeloma combining Venetoclax with Daratumumab has a good safety profile [11].

Here, Venetoclax and Daratumumab combination treatment resulted in a reduced leukemia growth in vitro and in vivo. Our data opens a potential avenue for novel Venetoclax drug combination with Daratumumab and supports further clinical investigation as a therapeutic approach in the treatment AML.

\section{Abbreviations}

AML: Acute myeloid leukemia; BCL-2: B-cell lymphoma 2; BM: Bone marrow: IP: Intra-peritoneal; MSC: Mesenchymal stromal cells; NSG: NOD.Cg-Prkdcscid IL2rgtm 1Wj//Sz;; PBS: Phosphate buffered saline

\section{Supplementary Information}

The online version contains supplementary material available at https://doi. org/10.1186/s40364-021-00291-y.

\section{Additional file 1:}

Additional file 2:

\section{Acknowledgements}

The work is supported by the Norwich Research Park (NRP), The Rosetrees Trust, The Big C, The NHS and The Wellcome Trust. The authors also thank Dr Allyson Tyler and Dr Karen Ashurst from the Laboratory Medicine department at the Norfolk and Norwich University Hospital for technical assistance. The authors wish to acknowledge the role of the NRP Biorepository in collecting and making available the samples used in the generation of this publication, and the patients who donated to the Biorepository. The authors also wish to thank the team at the Disease Modelling Unit of the University of East Anglia for assistance with the in-vivo studies. pCDH-LucferaseT2A-mCherry was kindly gifted by Professor Irmela Jeremias, MD, from Helmholtz Zentrum München, Munich, Germany.

\section{Authors' contributions}

J.J.M., K.M.B and S.A.R designed the research; J.J.M., C.H., A.L, J.A.M., and A.J., performed the research; J.J.M., S.A.R., and C.H. carried out in vivo work; K.M.B. and A.C provided essential reagents and knowledge. J.J.M., K.M.B. and S.A.R. wrote the paper. The author(s) read and approved the final manuscript.

\section{Funding}

JJM receives funding from the University of East Anglia and the Earlham Institute using support from the UK Research and Innovation (UKRI)
Biotechnology and Biological Sciences Research Council (BBSRC) under grants National Capability in Genomics and Single Cell "BBS/E/T/000PR9816. AL is funded by the University of East Anglia, CH by Wellcome Trust, JM by the Rosetrees Trust, AJ by The Big C. AC and KMB are supported by the Norwich and Norfolk University Hospital. SAR receives funding from the University of East Anglia. KMB and SAR also receive research funding from Abbvie and Jannsen.

\section{Availability of data and materials}

All data generated or analysed during this study are included in this published article.

\section{Declarations}

\section{Ethics approval and consent to participate}

Primary human tissue was taken following informed consent and under approval from the UK National Health Service Health Research Authority (LRECref07/H0310/146).

All animal work in this study was carried out in accordance with regulations set by the United Kingdom Home Office and the Animal Scientific

Procedures Act of 1986.

Consent for publication

Informed consent has been obtained from all patients (see above).

\section{Competing interests}

KMB and SAR receive research funding from Abbvie and Jannsen. The remaining author declare no competing financial interests.

\section{Author details}

${ }^{1}$ Norwich Medical School, University of East Anglia, Norwich Research Park, NR4 7UQ Norwich, UK. ${ }^{2}$ Earlham Institute, Norwich Research Park, NR4 7UH Norwich, UK. ${ }^{3}$ Department of Haematology, Norfolk and Norwich University Hospitals NHS Trust, Colney Lane, NR4 7UY Norwich, UK. ${ }^{4}$ Department of Molecular Haematology, Norwich Medical School, Norwich Research Park, NR4 7UQ Norwich, UK.

Received: 25 January 2021 Accepted: 29 April 2021

Published online: 13 May 2021

\section{References}

1. Shafat MS, Gnaneswaran B, Bowles KM, Rushworth SA. The bone marrow microenvironment - Home of the leukemic blasts. Blood Rev. 2017;31(5): $277-86$.

2. Vaux DL. Immunology. Ways around rejection. Nature. 1998;394(6689):133.

3. Tzifi F, Economopoulou C, Gourgiotis D, Ardavanis A, Papageorgiou S, Scorilas A. The Role of BCL2 Family of Apoptosis Regulator Proteins in Acute and Chronic Leukemias. Adv Hematol. 2012;2012:524308.

4. Campos L, Rouault JP, Sabido O, Oriol P, Roubi N, Vasselon C, et al. High expression of bcl-2 protein in acute myeloid leukemia cells is associated with poor response to chemotherapy. Blood. 1993;81(11):3091-6.

5. Pan R, Hogdal L, Benito JM, Bucci D, Han L, Borthakur G, et al. Selective BCL-2 inhibition by ABT-199 causes on-target cell death in acute myeloid leukemia. Cancer Discov. 2014;4(3):362-75.

6. DiNardo CD, Pratz KW, Letai A, Jonas BA, Wei AH, Thirman M, et al. Safety and preliminary efficacy of venetoclax with decitabine or azacitidine in elderly patients with previously untreated acute myeloid leukaemia: a nonrandomised, open-label, phase 1b study. Lancet Oncol. 2018;19(2):216-28. 
7. Ramsey HE, Fischer MA, Lee T, Gorska AE, Arrate MP, Fuller L, et al. A Novel MCL1 Inhibitor Combined with Venetoclax Rescues Venetoclax-Resistant Acute Myelogenous Leukemia. Cancer Discov. 2018;8(12):1566-81.

8. Naik J, Themeli M, de Jong-Korlaar R, Ruiter RWJ, Poddighe PJ, Yuan H, et al. CD38 as a therapeutic target for adult acute myeloid leukemia and T-cell acute lymphoblastic leukemia. Haematologica. 2019;104(3):e100-e3.

9. Mistry JJ, Moore JA, Kumar P, Marlein CR, Hellmich C, Pillinger G, et al. Daratumumab inhibits acute myeloid leukaemia metabolic capacity by blocking mitochondrial transfer from mesenchymal stromal cells. Haematologica. 2020:haematol.2019.242974.

10. Marlein CR, Piddock RE, Mistry JJ, Zaitseva L, Hellmich C, Horton RH, et al. CD38-Driven Mitochondrial Trafficking Promotes Bioenergetic Plasticity in Multiple Myeloma. Cancer Res. 2019;79(9):2285-97.

11. Kaufman JL, Baz RC, Harrison SJ, Quach H, Ho S-J, Vangsted AJ, et al. Updated analysis of a phase $1 / / 1$ study of venetoclax in combination with daratumumab and dexamethasone, +/- bortezomib, in patients with relapsed/refractory multiple myeloma. J Clin Oncol. 2020;38(15_suppl):8511-.

\section{Publisher's Note}

Springer Nature remains neutral with regard to jurisdictional claims in published maps and institutional affiliations.

Ready to submit your research? Choose BMC and benefit from:

- fast, convenient online submission

- thorough peer review by experienced researchers in your field

- rapid publication on acceptance

- support for research data, including large and complex data types

- gold Open Access which fosters wider collaboration and increased citations

- maximum visibility for your research: over $100 \mathrm{M}$ website views per year

At $\mathrm{BMC}$, research is always in progress.

Learn more biomedcentral.com/submissions 